\title{
Robotic Leg Design to Analysis the Human Leg Swing from Motion Capture
}

\author{
Riky Tri Yunardi*, Aji Akbar Firdaus, Eva Inaiyah Agustin \\ Department of Engineering, Faculty of Vocational, Universitas Airlangga Campus B UNAIR JI. Srikana 65, \\ 60286, Surabaya, Indonesia \\ ${ }^{*}$ Coressponding author, e-mail: rikytriyunardi@vokasi.unair.ac.id
}

\begin{abstract}
In this paper presented the prototype of robotic leg has been designed, constructed and controlled. These prototype are designed from a geometric of human leg model with three joints moving in $2 D$ plane. Robot has three degree of freedom using DC servo motor as a joint actuators: hip, knee and ankle. The mechanical leg constructed using aluminum alloy and acrylic material. The control movement of this system is based on motion capture data stored on a personal computer. The motions are recorded with a camera by use of a marker-based to track movement of human leg. Propose of this paper is design of robotic leg to present the analysis of motion of the human leg swing and to testing the system ability to create the movement from motion capture. The results of this study show that the design of robotic leg was capable for practical use of the human leg motion analysis. The accuracy of orientation angles of joints shows the average error on hip is $1.46^{\circ}$, knee is $1.66^{\circ}$, and ankle is $0.46^{\circ}$. In this research suggesting that the construction of mechanic is an important role in the stabilization of the movement sequence.
\end{abstract}

Keywords: robotic leg, human leg swing, motion capture, marker-based

\section{Introduction}

In the field of biomechanics simulation tools are widely used in robotics. The researcher use the simulator to validate their developed algorithms with real task performance. The simulator designed to capable of moving and repeating the movement with high accuracy. A robotic leg is one of the platform tool was designed to generate sequence of movement. In previous work. Robotic leg used pneumatic as actuator for robot to simulate legged locomotion by use the air pressure [1-2]. It was used latex rubber tube like a muscle were have a limitation of motion range to control of these actuators. To controlling of mechanical robot, DC motors has used to generate leg movement that requires small torque on light weight actuator [3-4].

Human motion analysis is the study of instrumentation for measuring body movements and part of body mechanics [5]. The human walking shows the pattern of movement of the human foot during gait that has repeated cycles. The walking step are describing a cycle between of leg stance phase and swing phase. The motion of the human leg is performed of the movement of human body parts that gives a motion information [6-8]. Motion capture is a algorithm used to get information of movement an object [9]. And to capture the movement use markers that mounted on positions, hip, knee and ankle on the human leg [10].

In this paper, we describe a design the robotic leg and controls the movements from motion capture to analysis the human leg swing. These prototype was designed from a geometric of human leg model for the movement to approach the human movement like leg swing during walking. The motion of robotic leg has 3 DOF (Degree of Freedom) and modeled with three joint actuators moving in 2D plane. Servo motor are mounted on joint the robotic leg that structure constructed by aluminum metal and acrylic material. Design control of the movements of a robotic leg is based on motion capture data stored in each leg for the hip, knee and ankle. Most area of the overview in the our research of robotics leg is shown in Figure 1.

The paper is structured as follows. Configuration of robotic leg is described in Section 2. In Section 3, shows the architecture control including inverse kinematics, robot controller and motion capture interface. In Section 4 presents the results and analysis followed by the conclusion in section 5. Acknowledgements are given in Section 6 and References. 


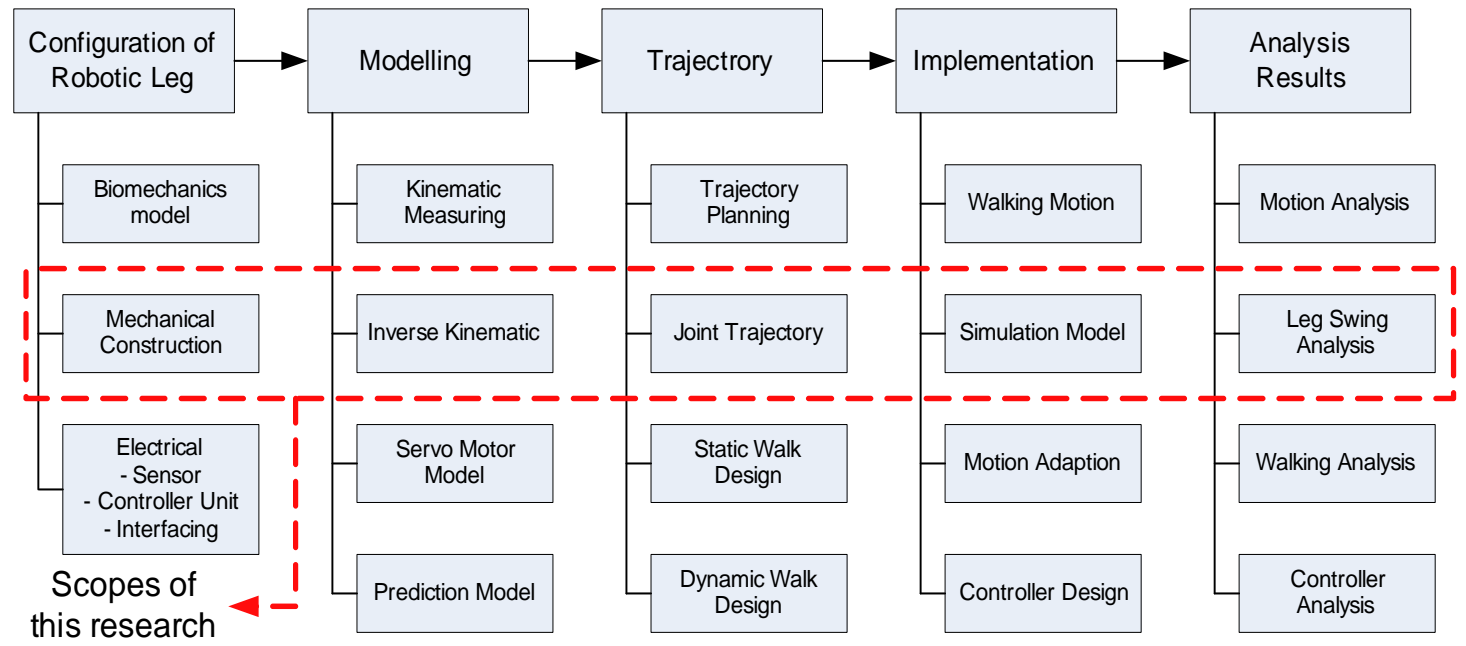

Figure 1. Overview of the research of robotics leg

\section{Configuration of Robotic Leg}

The configuration of robotic leg is modeled from the functional mobility of the human lower limb system and can represented as a sequence of links connected by joints [11]. The robotic leg mainly takes place in sagittal position which has three joint that is hip, knee and ankle and used servo motors as actuators to serve moving of joints.

\subsection{Robotic Leg Design}

The human body is a frame arrangement connected with the joints. The approach describes the human body from a rod shape to projected 2D image. The shape of a stick figure represents a simple movement on the bone used for the basic analysis of the gait. Human leg model used 4 line segments and 3 joints to represent the hip, knee, and ankle, shown in Figure 2. The model aimed to constructing a kinematic for gait analysis in human walking. The angle and joint displacement were measured from image sequences and kinematic pattern was detected for each walking cycle. The model consists of main three joints in left sides and the parameters of line segments and joints based on Denavit and Hartenberg method.
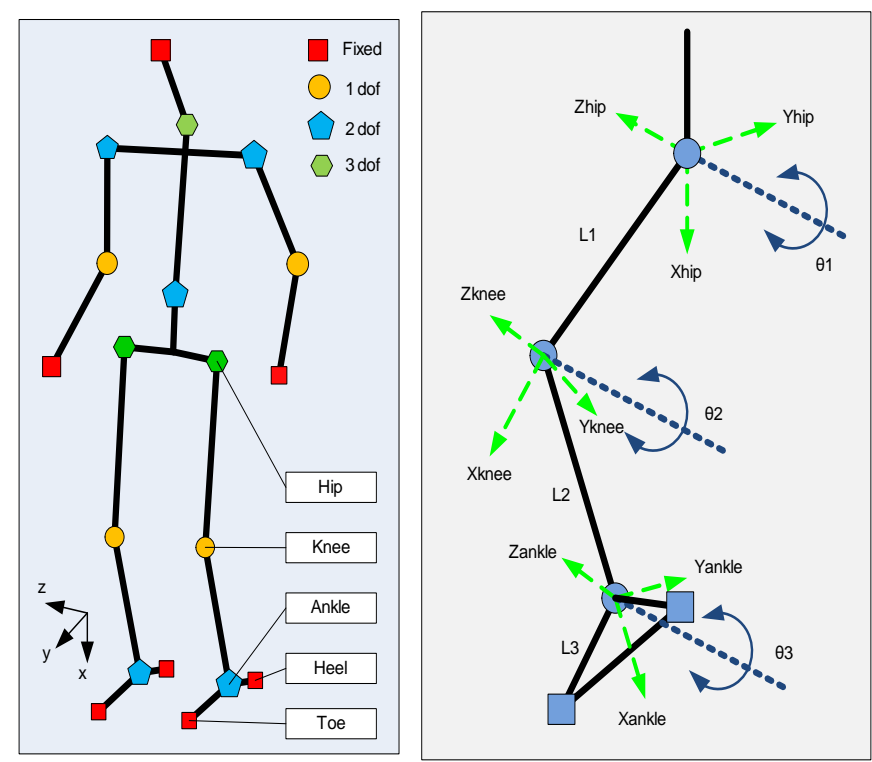

Figure 2. The stick figure model of the human body and the left leg 


\subsection{Robot Hardware}

In design harware of robotic leg, the mechanic joint has three degree of freedom. Each joints for hip, knee and ankle as shown in Figure 3. The weight of the robotic leg for simulator is must consider. Structure of mechanic is constructed by aluminum metal and acrylic material that light and durable. Servo motor are mounted on joint the robotic leg to serve its actuators movement. The first servo attached at hip and second at knee, distance between joints is 12 $\mathrm{cm}$. The second servo and the third servo attached at the knee are connected with a link that has a distance of $11 \mathrm{~cm}$. The third servo is connected with the toe as an end-effector with a distance of $3 \mathrm{~cm}$.
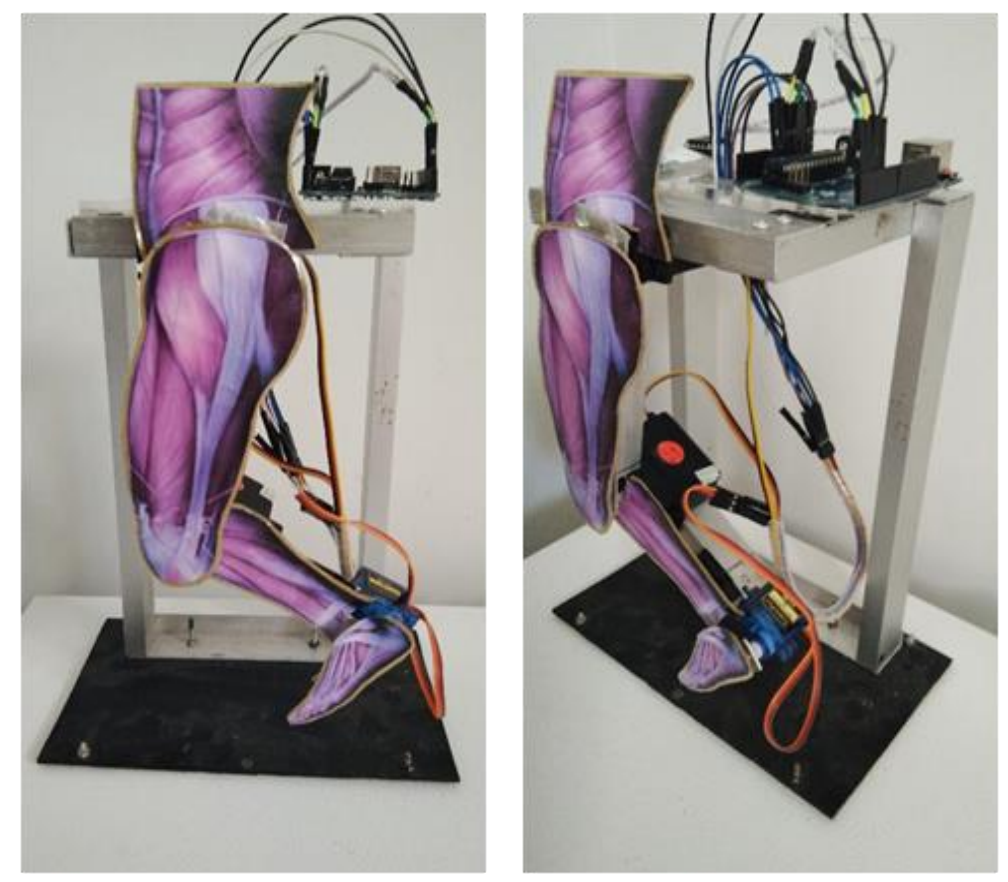

Figure 3. The hardware of robotic leg

\section{Control Architecture}

The control architecture is basically divided in three parts: 1 . Inverse kinematics used for computation the angles of the joints of the robotic arm, 2. Robot controller contains electronic moduls that needed by a controller to made decisions based on the desired values, And 3 . This part describes the software of motion capture interface using visual sensor.

\subsection{Inverse Kinematics}

Inverse kinematics is the process to get of the angles of the coordinates on the leg model desired in the Cartesian plane system. To obtain the formula of inverse kinematics that applied at a robotic leg, it necessary to use the Denavit and Hartenberg algorithm. Parameters obtained through the application of this method are displayed in Table 1. This leg model is formed by a structure composed of three segments (links) of fixed length. This robot is formed by a symmetrical structure composed of six identical legs, having three degrees of freedom (DOF) of rotational type in each leg. For getting the inverse kinematics equations is dependent on the configuration of robotic leg. In this research, through geometrical considerations based on the shape of leg shown in Figure 4.

Table 1. The Parameters of Denavit-Hartenberg

\begin{tabular}{ccccc}
\hline Link & $\mathrm{a}_{\mathrm{i}}$ & $\mathrm{a}_{\mathrm{i}}$ & $\mathrm{d}_{\mathrm{i}}$ & $\theta_{\mathrm{i}}$ \\
\hline 1 & $\mathrm{a} 1$ & $90^{\circ}$ & $\mathrm{d} 1$ & $\theta_{1}$ \\
2 & $\mathrm{a} 2$ & $0^{\circ}$ & 0 & $\theta_{2}$ \\
3 & $\mathrm{a} 3$ & $180^{\circ}$ & 0 & $\theta_{3}$ \\
\hline
\end{tabular}

Bulletin of EEI Vol. 6, No. 3, September 2017 : $256-264$ 


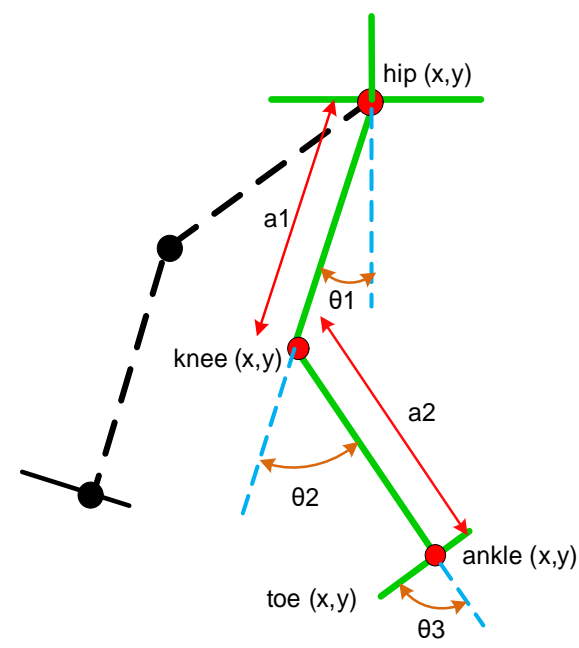

Figure 4. Planar robotic leg modeling

The kinematic model in the present study consists of four links to make an approximate characteristics of walking motion that similar of the lower human body. According to Figure 4, first joint unites with the body of the robot According to Figure 4, first joint is the point where the leg unites with the body of the robot is hip. The joint between the thigh and shank is called knee. And the ankle is connected with shank. A cycle of step in the gait of a leg consists of stance phase and swing phase. The robotic leg modeling must be consider during moving, especially when the foot place on the ground. So in experiments in these simulations and modeling, the dynamics effects are ignored.

In the proposed method, position of joints described on a Cartesian coordinate planes by 2 dimension pixel operation. The kinematic analysis of a leg robot model used three degree of freedom to derivate inverse kinematics functions by defining three value of rotation that are connected on each joint. To define the between rotation of joint and translation of link, the parameters of Denavit-Hartenberg are involved. Based on the equation (1) until (5), the transformation of each joint will be obtained with the forward kinematics functions. These functions define the ankle position according to angle of joint and the adjacent links.

$$
\begin{aligned}
& \text { toe }_{x}=a_{1} \cos \theta_{1}+a_{2} \cos \left(\theta_{1}+\theta_{2}\right)+a_{3} \cos \left(\theta_{1}+\theta_{2}+\theta_{3}\right) \\
& \text { toe }_{y}=a_{1} \sin \theta_{1}+a_{2} \sin \left(\theta_{1}+\theta_{2}\right)+a_{3} \sin \left(\theta_{1}+\theta_{2}+\theta_{3}\right) \\
& \varnothing=\theta_{1}+\theta_{2}+\theta_{3} \\
& \text { ankle }_{x}=\text { toe }_{x}-a_{3} \cos \emptyset \\
& \text { ankle }_{y}=\text { toe }_{y}-a_{3} \sin \emptyset
\end{aligned}
$$

On the equation (6) and (7) using cosine rule, $\theta 1$ and $\theta 2$ can be obtained, these functions are used to determine angle of joint according with ankle and knee position.

$$
\begin{aligned}
& \theta_{2}=\cos ^{-1}\left(\frac{a_{n k l e_{x}}{ }^{2}+a_{n k l e_{y}}{ }^{2}-a_{1}{ }^{2}-a_{2}{ }^{2}}{2 a_{1} a_{2}}\right) \\
& \theta_{1}=\tan ^{-1}\left(\frac{a_{n} k l e_{y}}{\text { ankle }_{x}}\right)-\tan ^{-1}\left(\frac{a_{2} \sin \theta_{2}}{a_{1}+a_{2} \cos \theta_{2}}\right)
\end{aligned}
$$

And the value of $\theta_{3}$ is can be obtained as equation (8).

$$
\theta_{3}=\varnothing-\theta_{1}-\theta_{2}
$$




\subsection{Robot Controller}

The movement of links is held at the cartesian space in the joint space, which requires the integration of the inverse kinematic. The movements of a robotic leg is based on motion capture data stored. Controller contains electronic moduls that needed for controlling the mechanism to the desired values of joint rotation. In motion capture unit, movements of the mounted markers on leg are captured using camera. The tracking system capturing the marker in many times per second to identify the motion the positions or angles between the markers. Afterwards all joint angles are calculated by kinematics model on the 2 dimension plane.

The microcontroller that used for convert the joint angles data from motion capture unit to servo controller pulses is handled by ATmega328. The PWM (pulse witdh modulation) trigger for the servo motor is generated by the servo controller. The mechanism of robotic leg is driven by three servo motors mounted on each joint. The general robotic leg control architecture is shown in Figure 5.

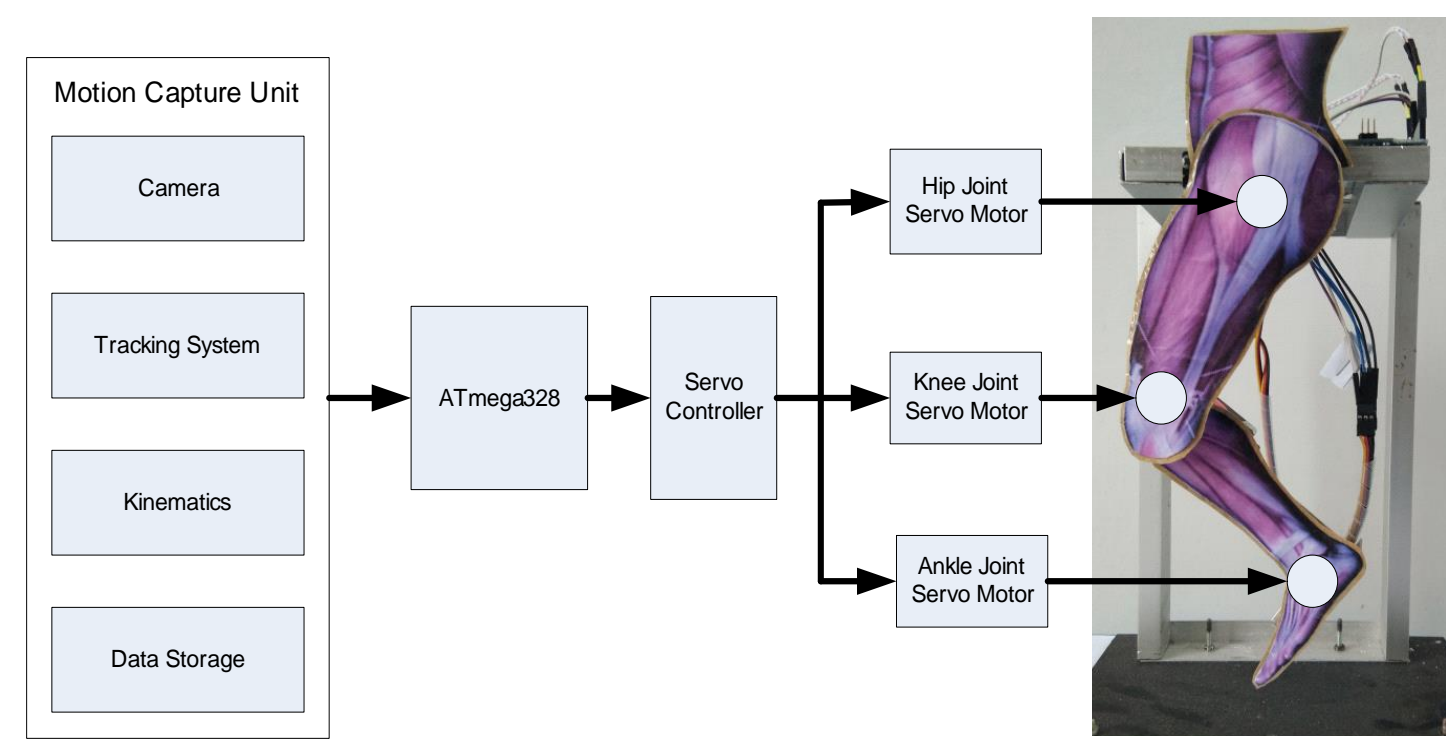

Figure 5. Robotic leg control architecture

\subsection{Motion Capture Interface}

We constructed the motion capture interface, which is being used for marker recognition and tracking of gait sequences shown in Figure 6. The motion capture interface captured the marker on each joint and estimated by calculating its centroid location. This interface can tracking of 3 DOF for each time step and graphically displays the human leg movement in sagittal view. The tool also allows the playback of an iteration of entered gait sequence and allows the controlling of the hardware of robotic leg that downloaded to the controller unit. In this study, the motion capture interface does not perform for analysis of accuracy the mechanics to a gait. 


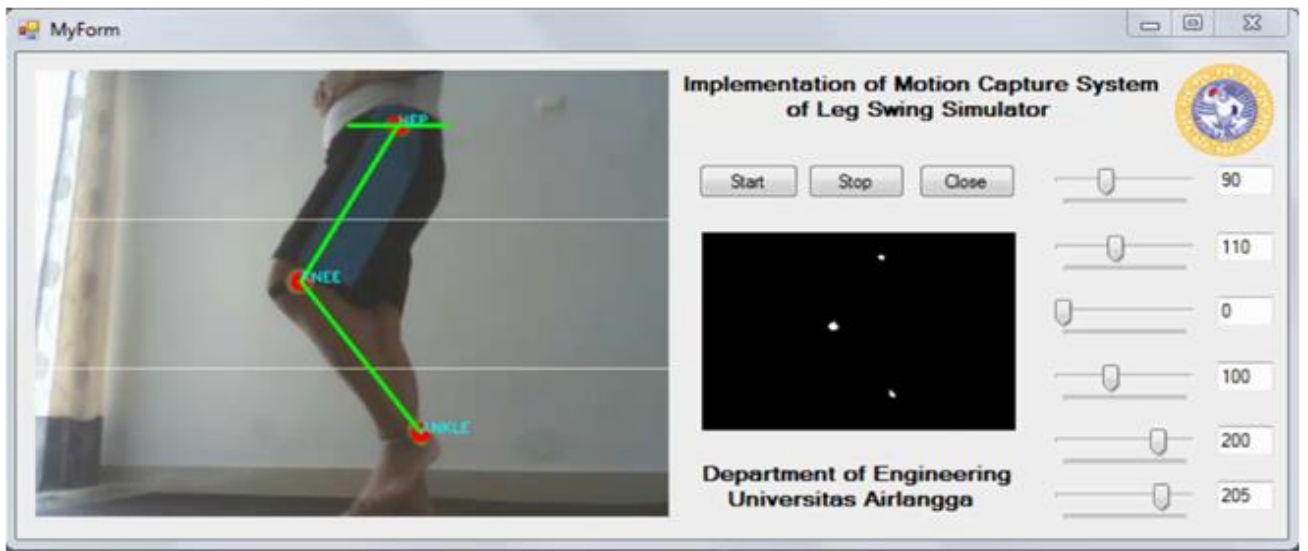

Figure 6. Motion capture interface tool

\section{Results and Discussions}

\subsection{Marker-based Data Processing}

In this project was developed the marker-based motion capture system. This system Implemented for capable of calculating kinematic of leg model from markers that mounted at the leg using camera. The frame rate of camera up to 30 frames per second with 24 bit RGB format. Personal computer are used to calculation process to get the data of joint rotations. Image processing are implemented for calculating and analyze are focus on $2 \mathrm{D}$ image by pixel operation. The system runs to get motion information and recording the motion into the database of walking leg movement. We tested the subject walking on a flat floor. The experiment held in under laboratory condition to minimize interference. The data iteration of the walking step for describing a cycle of leg swing are acquired shown in Figure 7.
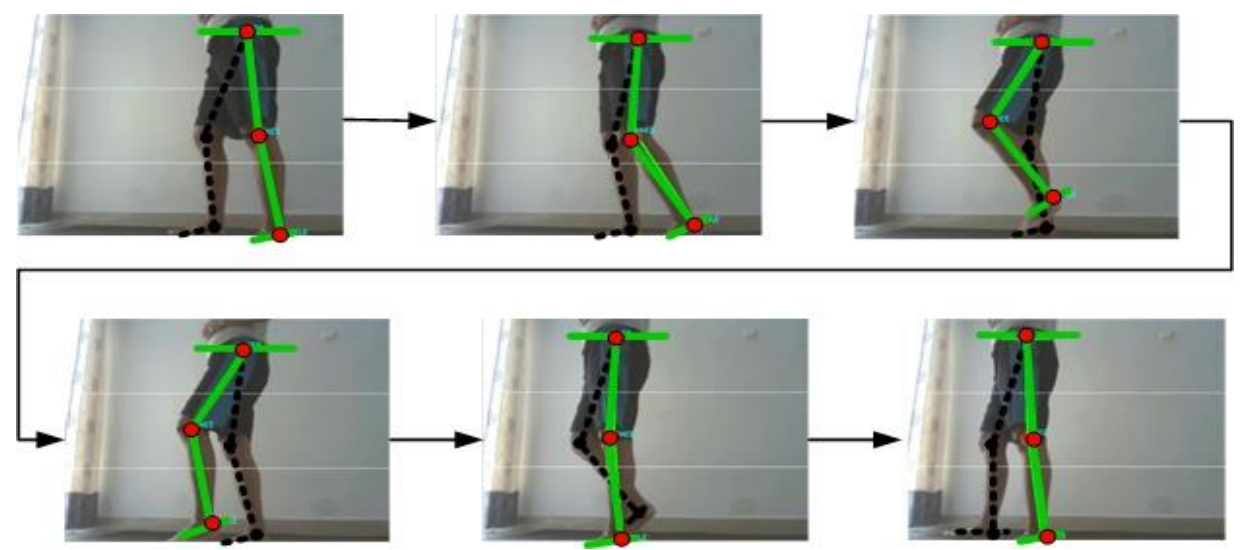

Figure 7. The markers captured by camera on the leg [9]

\subsection{Leg Swing Experiment on the Prototype of Robotic Leg}

The parameters of joint position in Cartesian space of the leg swing movement based on the database from motion capture. The coordinate of joint position data generated a an iteration of gait sequence of human leg motion. The joint angle trajectories on hip, knee and ankle are derived from the inverse kinematics model. To controlled joints of robotic leg, the servo controller unit and 3 servos are mounted on joint the prototype of robotic leg.

To test the implementation of proposed method, we also developed the hardware of robotic leg. The ability of the robotic leg is capable of moving like leg swing during walking. In the experiment, movement of system is created for analyzing the movement of the leg when 
walking in one cycle. Figure 8 is shown simulation of swing leg motion were performed by the prototype of robotic leg.
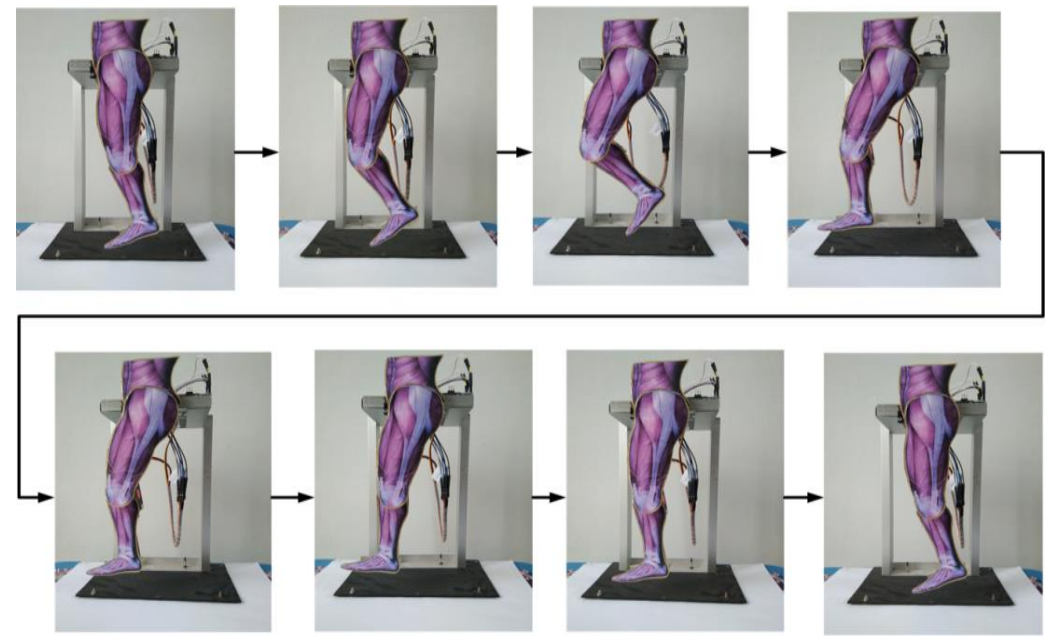

Figure 8. Leg swing simulation experiments [10]

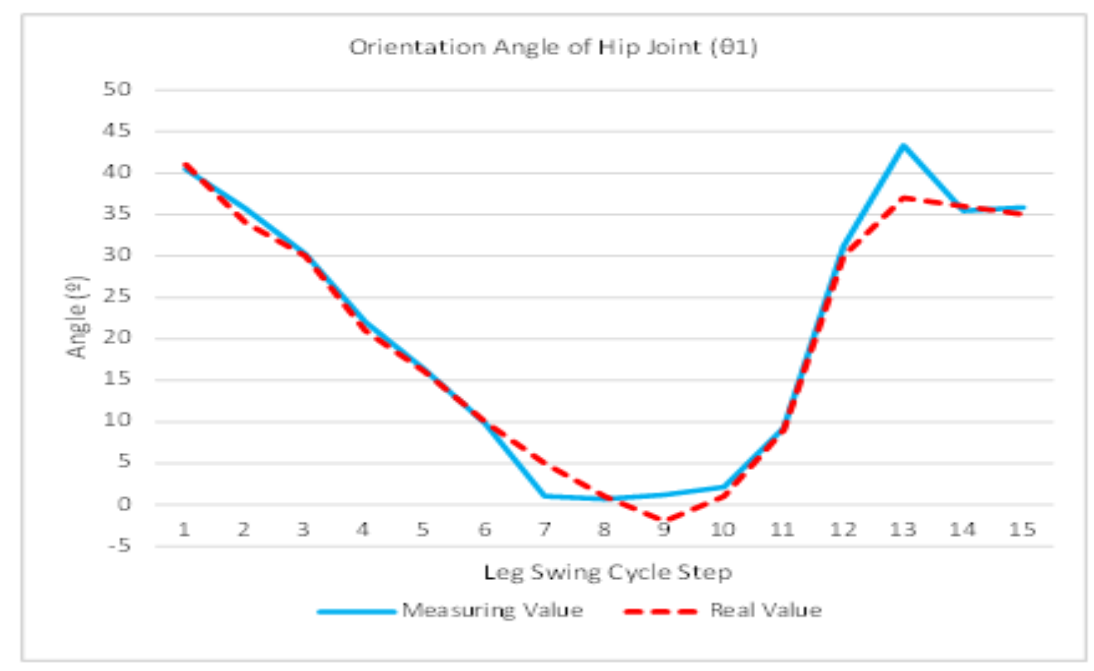

Figure 9. Performance of hip joint rotation on the robotic leg model

\subsection{Modeling and Simulation Performance}

In the design of robotic leg, servo motors as actuators are used to serve motion of its joints. The robotic leg with sagittal position has three joint of geometric human leg model that is hip, knee and ankle. The mechanics of system capable of movement of the foot swing from the position data of the joints in the Cartesian space by kinematics equation. To prove the performance of the design the robotic leg and the control the movements, data of joint rotations implemented into system to swing like a leg during walking. Modeling and simulation performances of joint rotation on the robotic leg model are shown in Figure 9 until Figure 12.

The experiment results shown testing the performances of joint rotation using our proposed method shows the accuracy of hip joint shows the average error of angle is 1.46\%. The accuracy of knee joint has average error is $1.66^{\circ}$. Also, ankle joint has $0.46^{\circ}$. Experiment on the design the robotic leg is capable of the foot swing movement. By considering in actual practice, development of system should considered the marker-less method and the construction of mechanic, for increase the accuracy of motion capture system and stabilization of the 
movement. It can be used as an alternative for simple motion capture system with applying of a human anatomical to allow application in the medical field to the human leg swing analysis.

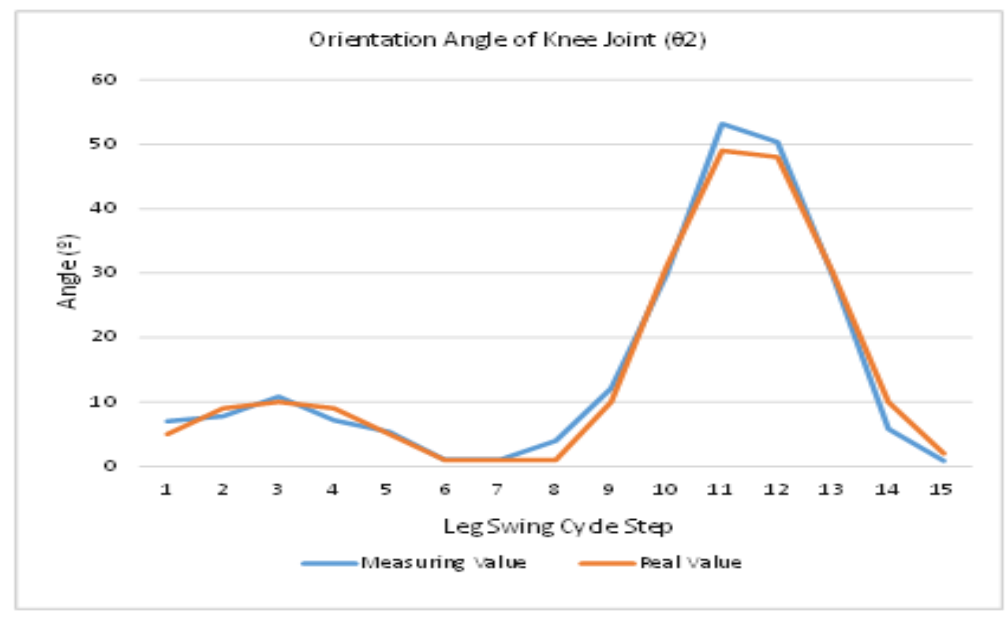

Figure 10. Performance of knee joint rotation on the robotic leg model

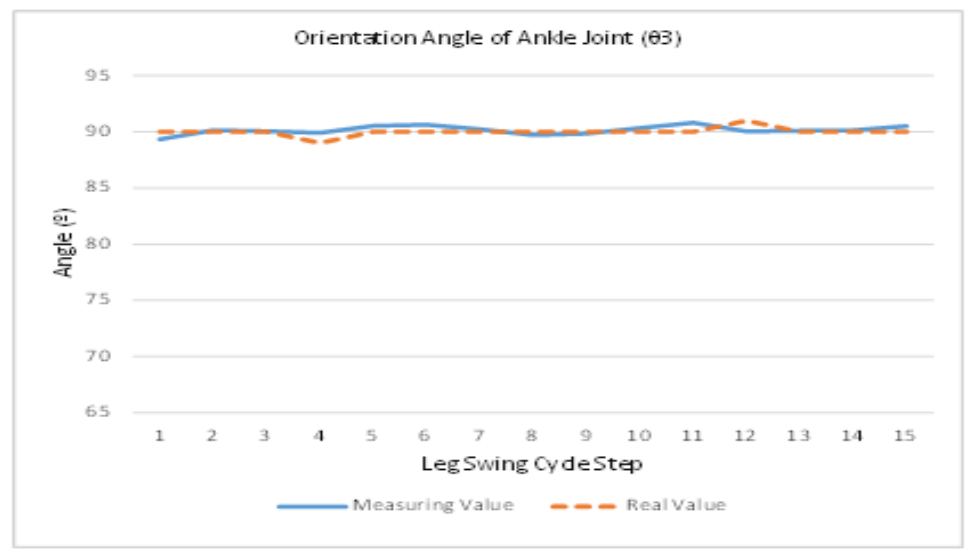

Figure 11. Performance of ankle joint rotation on the robotic leg model

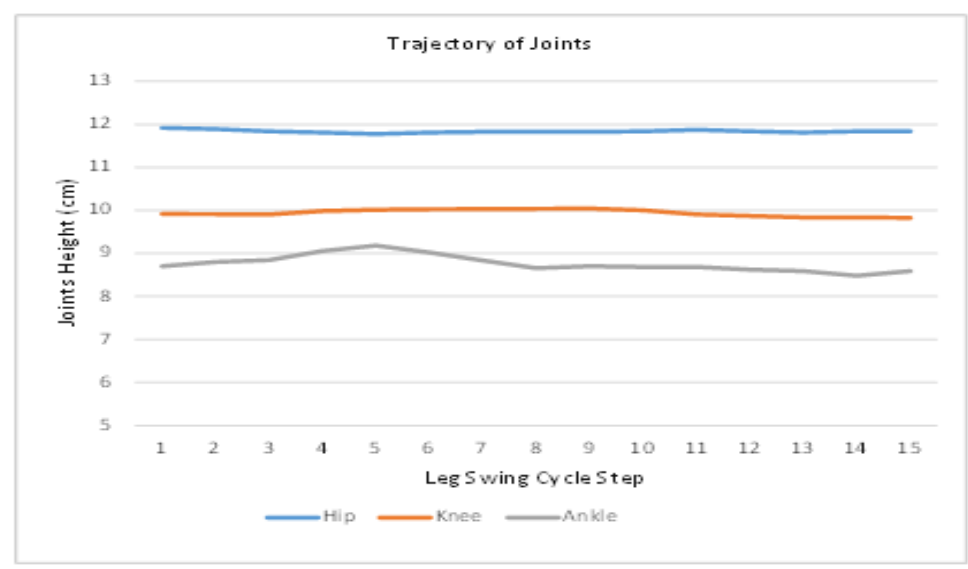

Figure 12. Trajectories of joint position on the robotic leg model 


\section{Conclusion}

In this research has been designed the prototype of robotic leg and controlled the movements from motion capture to analysis the human leg swing. These prototype using a model of geometric of human left leg. The robotic leg has three degree of freedom and used servo motor as a joint actuator on hip, knee and ankle. Control of the movements in 2D plane of a prototype are determined from the recorded based on motion capture data stored in each of joints. Data of the coordinate position that captured is using to control leg movement of joints. In the testing, the ability of the robotic leg to create the movement from motion capture are analyzed of the leg when swinging in one cycle. The sequence of movement are simulated to test the accuracy of orientation angles of joints on robotic leg. The accuracy of orientation angles of joints shows that the average error of hip is $1.46^{\circ}$, knee joint has average error is $1.66^{\circ}$, and ankle has $0.46^{\circ}$ of average error. The results of this study show that the construction of mechanic the real hardware is an important role in the design of robotic leg was capable of operating for analysis of the human leg motion

\section{Acknowledgements}

This work was supported by the financial funded No.19/UN3.1.14/KD/2017 by the Department of Engineering, Faculty of Vocational, Universitas Airlangga, Indonesia, is gratefully acknowledged. The author would like to say thank you to the researcher member that supporting this work.

\section{References}

[1] Colbrunn, Robb William, Gabriel M Nelson, Roger D. Quinn. Design and Control of a Robotic Leg with Braided Pneumatic Actuators. Intelligent Robots and Systems, 2001. Proceedings. 2001 IEEE/RSJ International Conference on IEEE 2001; 2.

[2] Chou, Ching-Ping, Blake Hannaford. Measurement and Modeling of McKibben Pneumatic Artificial Muscles. IEEE Transactions on Robotics and Automation. 1996; 12(1): 90-102.

[3] Zhao, Ye, et al. Phase Space Planning and Robust Control for Data-driven Locomotion Behaviors. Humanoid Robots (Humanoids), 2013 13th IEEE-RAS International Conference on. IEEE, 2013.

[4] Huang, Qiang, et al. Planning Walking Patterns for a Biped Robot. IEEE Transactions on Robotics and Automation. 2001; 17(3): 280-289.

[5] Lu, Tung-Wu, Chu-Fen Chang. Biomechanics of Human Movement and its Clinical Applications. The Kaohsiung Journal of Medical Sciences. 2012; 28(2): S13-S25.

[6] Maruyama, Tsubasa, et al. Motion-capture-based Walking Simulation of Digital Human Adapted to laser-scanned 3D as-is environments for Accessibility Evaluation.Journal of Computational Design and Engineering. 2016; 3(3): 250-265.

[7] Hienz, Hermann, Kirsti Grobel, Georg Offner. Real-time hand-arm Motion Analysis Using a Single Video Camera. Automatic Face and Gesture Recognition, 1996, Proceedings of the Second International Conference on. IEEE. 1996.

[8] Tiesel, Jan-Phillip, Jörn Loviscach. A Mobile Low-cost Motion Capture System Based on Accelerometers. Advances in Visual Computing. 2006: 437-446.

[9] Yunardi, Riky Tri, et al. Marker-Based Motion Capture for Measuring Joint Kinematics in Leg Swing Simulator. Instrumentation, Control and Automation (ICA), 2017 International Conference 2017.

[10] Yunardi, Riky Tri, et al. Implementation of Motion Capture System for Trajectory Planning of Leg Swing Simulator. The 2017 International Seminar on Sensors, Instrumentation, Measurement and Metrology. 2017.

[11] Lewis, M. Anthony, Theresa J. Klein. Robotic Biarticulate Muscle Leg Model. 2008. 